\title{
Guillain-Barré syndrome associated with herpes zoster
}

\author{
I.K. Hart and P.G.E. Kennedy \\ Department of Neurology, Institute of Neurological Sciences, Southern General Hospital, Glasgow G51 4TF, UK.

\begin{abstract}
Summary: A case of Guillain-Barré syndrome following herpes zoster is described. This is a rare association and the possible pathogenesis is discussed.
\end{abstract}

\section{Introduction}

Varicella zoster virus is a herpes virus which causes both varicella (chickenpox) and herpes zoster (shingles). Evidence suggests ${ }^{1}$ that during the viraemic phase of a primary varicella infection the virus reaches the skin and travels along the sensory axons to the dorsal root ganglia, where it can remain in a latent state for many years. In herpes zoster $(\mathrm{HZ})$ the virus is reactivated, often without an identifiable stimulus, and migrates down the sensory neurones to produce the characteristic painful vesicular eruption usually restricted to the cutaneous innervation of one or two dorsal root ganglia. The neurological complications of $\mathrm{HZ}$ can be classified as local or generalized. ${ }^{2}$ The local sequelae are (1) postherpetic neuralgia and (2) segmental or cranial nerve paresis. The generalized complications include (1) encephalitis, (2) cerebral vasculitis, (3) multi-focal leucoencephalitic syndrome and (4) Guillain-Barré syndrome (GBS). GBS resulting from $\mathrm{HZ}$ is rare and in some of the previously reported cases the association between the two conditions may have been fortuitous. Here we report a case of $\mathrm{HZ}$ closely followed by GBS.

\section{Case report}

A 74 year old man with a 13 year history of mechanical lower back pain developed left sided ophthalmic herpes zoster. Seven days later he reported sudden onset lumbar pain radiating to the right leg, accompanied by bilateral lower limb muscle cramps. After a further four days he experienced paraesthesiae and numbness in both feet. Over the next five days progressive symmetrical weakness of all four limbs developed together with an ascending sensory loss. His admission to a local hospital 12 days after the first neurological symptom was precipitated by a 48 hour history of increasingly severe respiratory distress, dysphagia and dysarthria. He had no bladder or bowel

Correspondence: I.K. Hart, B.Sc., M.R.C.P. Accepted: 16 July 1987 impairment and no history of injury, vaccination or exposure to toxins.

On examination he was apyrexial and normotensive with a sinus tachycardia of $130 /$ minute. Residual crusting of the skin in the distribution of the ophthalmic division of the left 5 th nerve was present. There was limitation of upward gaze and unsustained coarse nystagmus and bilateral lower motor neurone facial and bulbar weakness. Peak flow was $180 \mathrm{ml} / \mathrm{min}$. He had a flaccid symmetrical quadraparesis, MRC grade $3 / 5$ proximally and $2 / 5$ distally. All deep tendon reflexes and both plantar responses were absent. Appreciation of vibration, joint position and pinprick was impaired distally in the limbs with a complete anterior and posterior sensory level to pinprick at T4.

On investigation, urea, electrolytes, liver function tests, serum porphyrins, immunoelectrophoresis and full blood count were normal. Chest X-ray was unremarkable and full length myelogram revealed bulging of $L 4$ and $L 5$ vertebral discs with non-filling of the $\mathrm{L} 4, \mathrm{~L} 5$ and $\mathrm{S} 1$ roots bilaterally. No abnormality of the dorsal or cervical regions was seen. The cerebrospinal fluid (CSF) was clear, colourless, and sterile on culture, with a total protein of $1.02 \mathrm{~g} / 1$, glucose $3.9 \mathrm{mmol} / 1$ and 17 nucleated cells $/ \mathrm{mm}^{3}$ - all lymphocytes. The IgG was $12.3 \%$ and IgG albumin index 0.65 . There were no oligoclonal bands. Nerve conduction studies confirmed a severe generalized demyelinating peripheral neuropathy with conduction velocities all below $25 \mathrm{~m} / \mathrm{s}$, prolonged distal latencies and proximal conduction block. Serological tests for syphilis and systemic lupus erythematosus were all negative. Paired sera taken 15 days apart revealed a rise in varicella zoster virus titres from $1 / 128$ to $1 / 256$. The CSF varicella zoster virus titre was less than 4 . There was no serological evidence of legionella, mycoplasma, Epstein-Barr virus, cytomegalovirus, herpes simplex, enterovirus or hepatitis A or B infection.

Over the next ten days, 6 plasma exchanges, each of 2.5 litres, were carried out. Two weeks after starting treatment the patient was breathing spontaneously and his multiple cranial nerve palsies had resolved, 
although his limb muscles remained weak. Limb power continues to improve slowly.

\section{Discussion}

Our case showed the typical clinical features of GBS consisting of areflexia and symmetrical progressive weakness of the limbs, respiratory and cranial musculature. ${ }^{3}$ Paraesthesiae and numbness of the feet in association with pain in the back and limbs is also well recognized. The presence of a distinct sensory level is atypical but does not exclude a diagnosis of GBS. A sensory level should always suggest spinal cord compression or myelitis, which is also a recognized complication of HZ. However in this case, there was no bladder or bowel upset and no other signs to indicate cord involvement. Moreover, myelography was normal and CSF studies and electrophysiological findings were fully consistent with the diagnosis of GBS.

In GBS there is an identifiable precipitant in 60$70 \%$ of cases. ${ }^{4}$ Preceding non-specific viral infection is found in $40-50 \%$ of cases and in about $10 \%$ there is a recognizable infection by agents such as mycoplasma, Epstein-Barr virus or hepatitis A. GBS following HZ is rare. Since the first description in $1924^{5}$ there have been only 24 reported cases. ${ }^{6,7,8}$ In some of these the causal nature of the association is unclear. One case ${ }^{5}$ had a positive Wasserman reaction and cardiovascular syphilis proven at post-mortem. The latent interval between onset of rash and neurological symptoms was seven days in our case. In the other cases this interval varied from two days to two months. ${ }^{7}$ Where longer latencies exist the association may simply have been fortuitous as both $\mathrm{HZ}$ and GBS are relatively common disorders.

The previous reports of post-zoster GBS reveal that the initial eruption can occur in any part of the body and the neurological symptoms can appear acutely or subacutely. There appears to be an inverse correlation between the length of the latent interval and the severity of the symptoms, short intervals being associated with a more severe illness and long intervals, more than two weeks, being followed by milder symptoms. ${ }^{6}$ Almost all cases have occurred after the age of 50 and there is an equal sex incidence. Mortality has been high at $\mathbf{5 0 \%}$, but this is probably due to the high median age of the reported group and the fact that most occurred before the recent advances and availability of intensive care.

The pathogenesis of post-zoster demyelinative polyneuropathy and the other widespread complications of $\mathrm{HZ}$ is poorly understood. GBS is thought to be due to an immune response directed against myelin in peripheral nerves. ${ }^{9}$ The possibility that humoral factors are involved provides the rationale for the use of plasma exchange. ${ }^{10}$ It seems likely that in $\mathrm{HZ}$ the syndrome is also immune-mediated in view of the latent period between the rash and the neurological symptoms, the close clinical and electrophysiological correlation with other cases of GBS and the histological demonstration of peripheral nerve demyelination in one fatal case. ${ }^{6}$ The very uncommon occurrence of GBS after $\mathrm{HZ}$ suggests that most individuals with $\mathrm{HZ}$ are in some way able to suppress an autoimmune reaction. However $\mathrm{HZ}$ itself is $5-25$ times more common in immunosuppressed patients ${ }^{11}$ and, as far as we are aware, no case followed by the GBS has been reported in such circumstances. It is possible that the frequency of this syndrome is underestimated and that careful examination of patients with $\mathrm{HZ}$ may reveal mild cases.

\section{Acknowledgements}

The help of our neurosurgical and neuroanaesthetic colleagues is warmly appreciated.

\section{References}

1. Hope-Simpson, R.E. The nature of herpes zoster: A long term study and a new hypothesis. Proc Roy Soc Med 1965, 58: 9-20.

2. Kennedy, P.G.E. Neurological complications of varicella - zoster virus In Kennedy, P.G.E. \& Johnson, R.T. (eds) Infections of the Nervous System. Butterworths, London, in press.

3. Asbury, A.K. Diagnostic considerations in the GuillainBarré syndrome. Ann Neurol 1981, Suppl. 9: 1-5.

4. Hughes, R.A.C. Acute inflammatory polyneuropathy. Br J Hosp Med 1978, 20: 688-693.

5. Wohlwill, F. Zur pathologischen Anatomie des Nervensystems beim herpes zoster. $Z$ ges Neurol Psych 1924, 89: $171-212$.

6. Knox, J.D.E., Levy, R. \& Simpson, J.A. Herpes zoster and the Landry-Guillain-Barré syndrome. $J$ Neurol Neurosurg Psych 1961, 24: 167-172.

7. Dayan, A.D., Ogul, E. \& Graveson, G.S. Polyneuritis and herpes zoster. J Neurol Neurosurg Psych 1972, 35: 170-175.

8. Gardner-Thorpe, C., Foster, J.B. \& Barwick, D.D. Unusual manifestations of herpes zoster. J. Neurol Sci 1976, 28: 427-447.

9. Cook, S.D., Dowling, P.C., Murray, M.R. \& Whittaker, J.W. Circulating demyelinating factors in acute idiopathic polyneuropathy. Arch Neurol 1971, 24: 136144.

10. Guillain-Barré Syndrome Study Group. Plasmapheresis and acute Guillain-Barré syndrome. Neurology 1985, 35: 1096-1104.

11. Dolin, R., Reichman, R.C. \& Mazur, M.H. Herpes zoster-varicella infections in immunosuppressed patients. Ann Int Med 1978, 89: 375-379. 